\title{
Local magnetic anisotropy by polarized neutron powder diffraction: Application of magnetically induced preferred crystallite orientation
}

\author{
I. A. Kibalin ${ }^{1,2}$ and A. Gukasov ${ }^{1, *}$ \\ ${ }^{1}$ Laboratoire Léon Brillouin, CEA-CNRS, CE-Saclay, 91191 Gif-sur-Yvette, France \\ ${ }^{2}$ PNPI NRC “Kurchatov Institute” Orlova Rosha, Gatchina, 188300 Leningrad Region, Russia
}

(Received 6 August 2019; published 13 November 2019)

\begin{abstract}
Polarized neutron diffraction allows one to determine the local susceptibility tensor on the magnetic site both in single crystals and powders. It is widely used in the studies of single crystals, but it is still hardly applicable to a number of highly interesting powder materials, such as molecular magnets or nanoscale systems because of the low luminosity of existing instruments and the absence of appropriate data analysis software. We show that these difficulties can be overcome by using a large area detector in combination with the two-dimensional Rietveld method and powder samples with a magnetically induced preferred crystallite orientation. This is demonstrated by revisiting two test powder compounds, namely, the low anisotropy (soft) ferrimagnetic compound $\mathrm{Fe}_{3} \mathrm{O}_{4}$ and the spin-ice compound $\mathrm{Ho}_{2} \mathrm{Ti}_{2} \mathrm{O}_{7}$ with high local anisotropy. The values of the magnetic moments in $\mathrm{Fe}_{3} \mathrm{O}_{4}$ and the susceptibility tensors of $\mathrm{Ho}_{2} \mathrm{Ti}_{2} \mathrm{O}_{7}$ at various temperatures and fields were found in perfect agreement with these found earlier in single-crystal experiments. The magnetically induced preferred crystallite orientation was used to study the local susceptibility of a single-molecule magnet $\mathrm{Co}\left(\left[\left(\mathrm{CH}_{3}\right)_{2} \mathrm{~N}\right]_{2} \mathrm{CS}\right)_{2} \mathrm{Cl}_{2}$. Hence, the studies of local magnetic anisotropy in powder systems might now become accessible.
\end{abstract}

DOI: 10.1103/PhysRevResearch.1.033100

\section{INTRODUCTION}

Polarized neutron diffraction (PND), also called the "flipping ratio method," is a powerful tool to investigate intraor intermolecular magnetic interactions. It gives direct access to the magnetization distribution in the unit cell [1], permits separating the spin and orbital contributions [2], and allows for the determination of the local susceptibility tensor on the magnetic sites [3]. The magnetization distribution has contributed to the understanding of magnetic interactions by revealing the spin delocalization, the spin density distribution, and the wave functions of unpaired electrons [4]. In turn, the local susceptibility approach has been successfully used in recent studies of field-induced magnetic order in $R_{2} \mathrm{Ti}_{2} \mathrm{O}_{7}$ pyrochlore compounds with either uniaxial or planar anisotropy [5,6]. PND is becoming a reference in mapping the magnetic anisotropy at the atomic scale in molecular magnets [7,8]. Unfortunately, PND currently applies only to single crystals, which makes it inadequate for a number of highly interesting topics due to the difficulties encountered in growing sufficiently large samples.

Motivated by challenging scientific subjects, several attempts have been performed to investigate magnetized powder samples with polarized neutrons [9-12]. This allowed one

\footnotetext{
*arsen.goukassov@cea.fr

Published by the American Physical Society under the terms of the Creative Commons Attribution 4.0 International license. Further distribution of this work must maintain attribution to the author(s) and the published article's title, journal citation, and DOI.
}

to reveal magnetic moments of iron at different crystallographic sites in Prussian blue [10] and in $\alpha-\mathrm{Fe}_{16} \mathrm{~N}_{2}$ nanoparticles [11], as well as to amend the magnetic structure of highly anisotropic $\mathrm{TbCo}_{2} \mathrm{Ni}_{3}$ [12]. The validity of the method was illustrated by measurements of magnetic anisotropy in a polycrystalline sample of $\mathrm{Tb}_{2} \mathrm{Sn}_{2} \mathrm{O}_{7}$ [13]. As a proof of concept the local susceptibility parameters of $\mathrm{Tb}$ were found by a two-step procedure. First, the integrated intensities of the spin-up and spin-down components $I_{+}(h k l)$ and $I_{-}(h k l)$ were obtained by profile matching from the corresponding powder patterns. Then, the program CHILSQ of the Cambridge Crystallography Subroutine Library [14] was used to fit the integrated intensities. It is clear that such a procedure of data treatment can be applied only to highly symmetric crystal structures with a small unit cell. For more complex structures a Rietveld method needs to be developed.

A Rietveld analysis has become mandatory in powder diffraction for nuclear and magnetic structure refinements [15-17]. It refines various metrics, including lattice, structure, and magnetic parameters, and a preferred orientation to derive a calculated diffraction pattern. Once the calculated pattern becomes nearly identical to an experimental one, various properties pertaining to that sample can be obtained. However, the Rietveld method for polarized neutron powder diffraction (PNPD) has not yet been implemented. In the abovementioned polarized powder experiments special software programs (model dependent) were developed for the data treatment.

We note that at first PNPD measurements were performed on conventional powder diffractometers equipped with onedimensional (1D) detectors while modern unpolarized neutron powder diffractometers (Super-D2B, D20, SPODI) at reactor 
sources are equipped with two-dimensional (2D) detectors. Area detectors increase the efficiency of the instrument by an order of magnitude, but the common approach at these instruments consists in reducing the accumulated 2D data from the area detector into a 1D diffraction pattern by "unbending" the measured Debye cones. The resulting pattern is then treated using standard 1D Rietveld refinement [15-17]. Most recent powder diffractometers at advanced neutron spallation sources (WISH, POWGEN) use very large area detectors and operate in the time-of-flight (TOF) mode. This generates rather complex three-dimensional (3D) angular- and wavelength-dispersive data which are eventually transformed into a one-dimensional diffraction pattern $I(2 \theta)$ [or $I(\lambda)][18]$ to allow standard Rietveld refinement. It has been noted that a two-dimensional extension of the Rietveld method for neutron TOF powder diffraction taking into account the variation of the diffraction angle $2 \theta$ and wavelength $\lambda$ decreases the number of data-reduction steps and avoids the loss of highresolution information [19], but full-scale multidimensional Rietveld software for neutron TOF powder diffraction still needs to be developed.

Since area detectors increase considerably the efficiency of the instruments, we performed our PNPD measurements on diffractometers equipped with large 2D position-sensitive detectors. When using area detectors, the polarized neutron scattering is a function of $2 \theta$ and $\varphi$ but also of the angle between the magnetic field and the scattering vector. Moreover, neutrons are sensitive only to the magnetic moment perpendicular to the scattering vector. Therefore, the variation of intensity along the Debye cones can be used for the separation of nuclear and magnetic scattering contributions. For these reasons the transformation of angular-dispersive polarized neutron data from area detectors into one-dimensional $2 \theta$ pattern is not applicable. We note as well that the equation for powder averaging derived in the paper [13] is valid only for the vertical field and scattering in the horizontal plane. Here, we give an expression for powder averaging that is valid for general scattering geometry, which allows an implementation of the full scale 2D Rietveld method in PNPD.

Another possibility of increasing the efficiency of PNPD consists in using a magnetically induced preferred crystallite orientation. This technique can be applied to biaxial crystals in which the magnetic susceptibility tensor has different principal values (i.e., orthorhombic, monoclinic, and triclinic systems) $[20,21]$. Under a strong magnetic field the crystallites overcome the steric hindrance of powder packing and align their easy magnetization axis parallel to the applied magnetic field, leading to a crystallite preferred orientation. As a consequence, different reflections with similar Bragg angles $2 \theta$ appear at different angles along the Debye cones. No overlapping of these reflections occurs, which allows one to use diffractometers with a low resolution (hence, high luminosity) for powder diffraction.

Here, we show that the combination of a large area detector with 2D Rietveld analysis and magnetically induced preferred crystallite orientation enables PNPD in systems not available as single crystals. We illustrate this by the results of two test cases of magnetic materials: the low anisotropy (soft) ferrimagnetic compound $\mathrm{Fe}_{3} \mathrm{O}_{4}$ and the spin-ice compound $\mathrm{Ho}_{2} \mathrm{Ti}_{2} \mathrm{O}_{7}$ with high local anisotropy. We show that in both

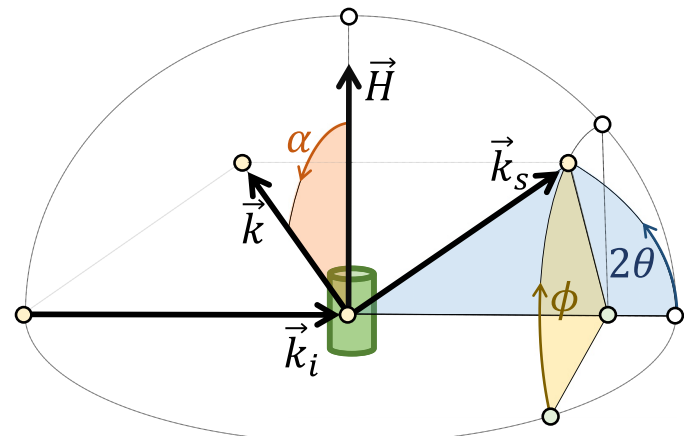

FIG. 1. The principal scheme of the scattering at the polycrystalline sample. A notation is explained in the text.

cases the combination of an area detector with the 2D Rietveld method shortens the acquisition time by an order of magnitude, without losing the precision of the parameter evaluation. Finally, we present the results of the local susceptibility studies on the single-molecule magnet $\mathrm{Co}\left(\left[\left(\mathrm{CH}_{3}\right)_{2} \mathrm{~N}\right]_{2} \mathrm{CS}\right)_{2} \mathrm{Cl}_{2}$ with a magnetically induced preferred orientation of the crystallites, which shows that the PNPD now opens large opportunities in the local anisotropy quantification of complex structures.

\section{POLARIZED NEUTRON POWDER DIFFRACTION}

It is well established that the flipping sum and difference of the integrated intensities $\left(I_{+}\right.$and $\left.I_{-}\right)$of polycrystalline samples are proportional to [1]

$$
\begin{gathered}
I_{+}+I_{-} \sim|N|^{2}+\left\langle\left|\vec{M}_{\perp}\right|^{2}\right\rangle, \\
I_{+}-I_{-} \sim N^{*}\left\langle\left(\vec{M}_{\perp} \cdot \vec{P}\right)\right\rangle+N\left\langle\left(\vec{M}_{\perp}^{*} \cdot \vec{P}\right)\right\rangle,
\end{gathered}
$$

where $N$ is the nuclear structure factor, and $\vec{M}_{\perp}$ is the projection of the magnetic structure factor $\vec{M}(\vec{k})$ perpendicular to the scattering vector $\vec{k} \cdot \vec{M}$ is induced by the magnetic field $\vec{H}$ applied in the vertical direction (Fig. 1) and $\vec{P}$ is the neutron polarization vector parallel to $\vec{H}$. Angle brackets show the powder averaging over scattering crystallites.

In soft magnetic materials the atomic magnetic moments $\vec{M}_{a}$ are directed along the applied field $\vec{H}$. Thus, the powder averaging of $\left|\vec{M}_{\perp}\right|^{2},\left(\vec{M}_{\perp} \cdot \vec{P}\right)$ can be written as

$$
\left\langle\left|\vec{M}_{\perp}\right|^{2}\right\rangle=\left|\sum_{a} M_{a} \sin \alpha f_{a}(\vec{k}) \exp \left[2 \pi i \vec{k} \cdot \vec{r}_{a}\right]\right|^{2}
$$

and

$$
\left\langle\left(\vec{M}_{\perp} \cdot \vec{P}\right)\right\rangle=\sum_{a} M_{a} P \sin ^{2} \alpha f_{a}(\vec{k}) \exp \left[2 \pi i \vec{k} \cdot \vec{r}_{a}\right],
$$

where the sum over $a$ includes all atoms in the unit cell with radius vector $\vec{r}_{a}$, and $f_{a}(\vec{k})$ is the magnetic form factor in the spherical approximation [22].

For paramagnets and diamagnets the structure factor $\vec{M}$ can be written as [13]

$$
\vec{M}(\vec{k})=\sum_{a} \frac{1}{N_{a}} f_{a}(\vec{k}) \sum_{p} R_{p} \chi_{a} R_{p}^{-1} \vec{H} e^{2 \pi i \vec{k}\left(R_{p} \vec{r}_{a}+\vec{t}_{p}\right)},
$$


where the sum over $a$ includes all independent atoms, and the sum over $p$ includes those generated from atom $a$ by the $N_{g}$ symmetry operators $\left\{R_{p}: \vec{t}_{p}\right\}$ of the space group $\mathcal{G} . N_{a}$ is the number of operators $q$ in $\mathcal{G}$ for which $R_{q} \vec{r}_{a}+\vec{t}_{q}=\vec{r}_{a} ; N_{g} / N_{a}$ is the multiplicity of the site $a$ which has point symmetry $Q_{a}$ generated by the rotational parts of the operators $q$. This implies that the local susceptibility tensor $\chi_{a}$ is invariant to the rotations in $Q_{a}$ so that $R_{q} \chi_{a} R_{q}^{-1}=\chi_{a}$ for all $R_{q}$ in $Q_{a}$. The number of independent components of the tensor $\chi_{a}$ varies from two for uniaxial site symmetries to six for triclinic ones.

The structure factor tensor $\chi(\vec{k})$, which is independent of the magnitude and direction of the applied field, can be expressed as follows,

$$
\chi(\vec{k})=\sum_{a} \frac{1}{N_{a}} f_{a}(\vec{k}) \sum_{p} R_{p} \chi_{a} R_{p}^{-1} e^{2 \pi i \vec{k}\left(R_{p} \vec{r}_{a}+\vec{t}_{p}\right)} .
$$

The expression for the powder averaging of $\left|\vec{M}_{\perp}\right|^{2}$ and $\left(\vec{M}_{\perp} \cdot \vec{P}\right)$ terms in the case of a magnetic field applied vertically and the detector in the horizontal plane has been given in Ref. [13]. It can be shown (see Supplemental Material [23]) that this expression can be generalized for any scattering geometry. Namely, the structure factor tensor is to be transformed into a Cartesian coordinate system with the $z$ axis parallel to the scattering vector. If the transformation is expressed through the matrix $T$ (see Supplemental Material [23]) the components of the tensor become $\Sigma=T \cdot \chi \cdot T^{-1}$ and the averaged terms above can be written as follows,

$$
\begin{aligned}
&\left\langle\left|\vec{M}_{\perp}\right|^{2}\right\rangle= \frac{1}{2} H^{2}\left[\left(\Sigma_{11}^{2}+2 \Sigma_{12}^{2}+\Sigma_{22}^{2}\right) \sin ^{2} \alpha\right. \\
&\left.+2\left(\Sigma_{13}^{2}+\Sigma_{23}^{2}\right) \cos ^{2} \alpha\right] \\
&\left\langle\left(\vec{M}_{\perp} \cdot \vec{P}\right)\right\rangle=P H\left(\frac{\Sigma_{11}+\Sigma_{22}}{2}\right) \sin ^{2} \alpha .
\end{aligned}
$$

Here, $\cos ^{2} \alpha=\cos ^{2} \theta \sin ^{2} \phi$. These equations describe the scattering along the Debye cones in the 2D Rietveld refinement. We note that for the special case of scattering in the equatorial plane $(\phi=0)$, expressions (7) and (8) are in exact accordance with these given before [13].

\section{A. 2D-diffraction profile}

In the two-dimensional case the calculated intensity $y_{ \pm}(2 \theta, \phi)$ for a single-phase diffraction pattern can be expressed for every data point by

$y_{ \pm}(2 \theta, \phi)=S \sum_{h} m_{h} L_{f} P_{h} I_{ \pm}(\alpha) \psi_{h}\left(2 \theta-2 \theta_{h}, \phi\right)+b(2 \theta, \phi)$,

where $S$ is a scale factor, $m_{h}$ is the multiplicity of reflection, $L(\theta, \phi)$ is the Lorentz factor, $P_{h}$ is the density of $(h k l)$ poles at the scattering vector (preferred orientation), $\psi_{h}\left(2 \theta-2 \theta_{h}, \phi\right)$ is the peak profile function normalized to unit area, and $b(2 \theta, \phi)$ is the background. The summation is done over all $h$ reflections for each data point. For a cylindrical detector [24] the Lorentz factor is $\sqrt{1-\sin ^{2} 2 \theta \sin ^{2} \phi} / \sin ^{2} \theta \cos \theta$. In the case of one-dimensional Rietveld refinement the profile function is usually described by the pseudo-Voight function. For the two-dimensional description of the diffraction pattern, an appropriate profile function still needs to be found. Here, we used the standard one-dimensional expression for the profile function $\psi_{h}\left(2 \theta-2 \theta_{h}\right)$ neglecting the dependence of the peak profile from the polar angle $\phi$. For these reasons the part of the diffraction pattern with a strong dependence of the peak profile from the polar angle $\phi$ was excluded from the refinement procedure.

It has been suggested $[9,11]$ that in the PNPD better quality information can be derived by using the flipping difference data, as contamination from the cryomagnet and the sample is largely eliminated in the difference. However, we note that a simultaneous refinement of the sum and the difference patterns is mandatory for the scaling of magnetic moment values. Moreover, as has been noted in Ref. [13], in the cases of strong magnetic scatterers with high anisotropy the sum patterns might contain a number of purely magnetic reflections which do not depend on neutron polarization.

\section{B. Experiment and data treatment}

Neutron diffraction studies were performed at the Orphée 14MW reactor of the Laboratory Léon Brillouin, CEA Saclay. The diffraction patterns were collected on the diffractometer $5 \mathrm{C} 1$, equipped by a position-sensitive detector with cylindrical geometry covering $80^{\circ}$ and $25^{\circ}$ in the horizontal and vertical directions, using neutrons of wavelength $\lambda=0.84 \AA$ obtained with a Heusler alloy monochromator. The incident beam polarization $P$ is 0.91 .

PNPD data were collected on powder sample of $\mathrm{Fe}_{3} \mathrm{O}_{4}$ in an external field of $0 \mathrm{~T}$ and $6 \mathrm{~T}$ below the Verwey transition (at $10 \mathrm{~K}$ ) and above it (at $150 \mathrm{~K}$ ). The experiments with a sintered powder sample of $\mathrm{Ho}_{2} \mathrm{Ti}_{2} \mathrm{O}_{7}$ were performed in the temperature range from 5 up to $50 \mathrm{~K}$ in a magnetic field of $1 \mathrm{~T}$.

Measurements of the $\mathrm{Co}$ (II) complex with single-molecule magnet behavior have been carried out on the thermal polarized neutron lifting counter diffractometer 6T2 (LLB-Orphée, Saclay). Neutrons of wavelength $1.4 \AA$ were monochromated by a vertically focusing graphite crystal and polarized by a supermirror bender. The polarization factor of the beam was 0.95 . The position-sensitive detector has a flat geometry. Data treatment was performed using the newly developed 2D Rietveld software RHOCHI [25].

\section{SOFT FERRIMAGNETIC $\mathrm{Fe}_{3} \mathrm{O}_{4}$}

Magnetite $\mathrm{Fe}_{3} \mathrm{O}_{4}$, as an original magnetic material with modern applications ranging from spintronics to magnetic resonance imaging (MRI) contrast agents was chosen as an example of a soft (low anisotropy) ferrimagnet for the software benchmarking. At ambient temperatures it orders in an inverted cubic spinel ferrite with the tetrahedral $(A)$ site occupied by $\mathrm{Fe}^{3+}$ ions and with $\mathrm{Fe}^{2+}$ and $\mathrm{Fe}^{3+}$ ions coexisting at the same octahedral $(B)$ site [26]. The magnetite undergoes a first-order transition below $120 \mathrm{~K}$ where the resistivity increases by two orders of magnitude and structural distortions from cubic symmetry occur [27,28]. It is suggested that this transition is driven by a charge ordering of $\mathrm{Fe}^{2+}$ and $\mathrm{Fe}^{3+}$ ions [29]. Polarized neutron diffraction measurements performed on a single crystal of magnetite earlier have shown 

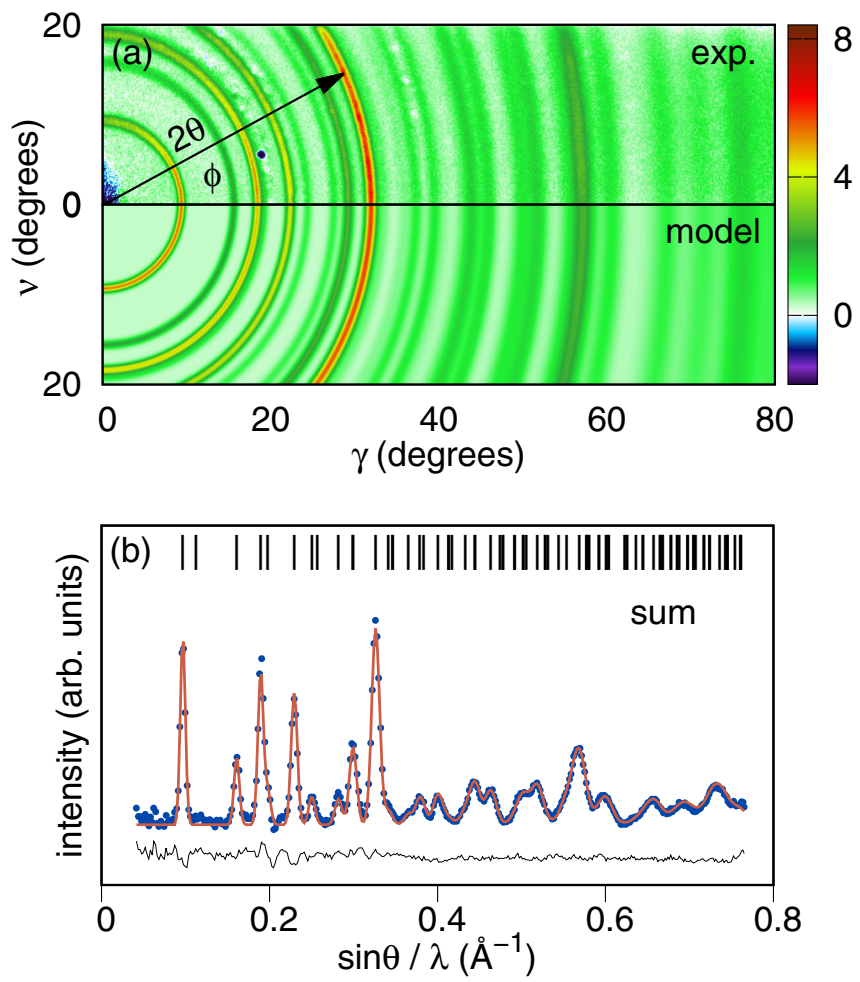

FIG. 2. Flipping sum diffraction patterns collected on $\mathrm{Fe}_{3} \mathrm{O}_{4}$ at $T=150 \mathrm{~K}, H=0 \mathrm{~T}$. The measured 2D pattern is shown on the top, the calculated is shown on the bottom (a), and chi squares normalized per number of points is 5.87. Diffraction profile estimated near the equatorial plane (b). $\gamma$ is the azimuthal angle and $v$ is the elevation angle in the laboratory coordinate system $(x y z)$, where $\vec{x}\|\vec{k}, \vec{z}\| \vec{H}$.

the antiparallel orientation of the moments at the tetrahedral and octahedral sites but surprisingly no difference between the magnetic moments at the sites was found [30].

In the absence of a magnetic field the flipping sum diffraction pattern corrected for the background is presented in Fig. 2 together with the standard 1D diffraction pattern limited to the equatorial plane. One can see that the scattering intensity distribution along the Debye cones is rather homogeneous and the width of the cones increases with the $\phi$ angle.

Figure 3 shows the $\phi$ dependence of the half width $H_{p V}$ and of the integrated intensity for $(111)\left(2 \theta_{h}=9.42^{\circ}\right)$ reflection. As seen from the figure, the width $H_{p V}$ remains approximately constant in the angular range from $0^{\circ}$ to $20^{\circ}$ and strongly increases at higher angles. Therefore the angular range from $0^{\circ}$ to $20^{\circ}$ was used in the refinement. In the meantime we note that the integrated intensity of the (111) reflection remains constant along the whole Debye cone, which is due to the fact that the magnetic moments are randomly oriented.

After refinement by the Rietveld method using Eqs. (3) and (4) the magnetic moments of iron in the tetrahedral and octahedral positions at $150 \mathrm{~K}$ and $0 \mathrm{~T}$ are found to be $-4.23(9) \mu_{B}$, $3.76(6) \mu_{B}$ for 1D data and $-4.09(2) \mu_{B}, 3.94(2) \mu_{B}$ for $2 \mathrm{D}$ ones. Different signs of the magnetic moments at the two sites correspond to their antiparallel orientation to each other. One can see that the values of the magnetic moments are in agreement for both refinements, while a significant decrease of

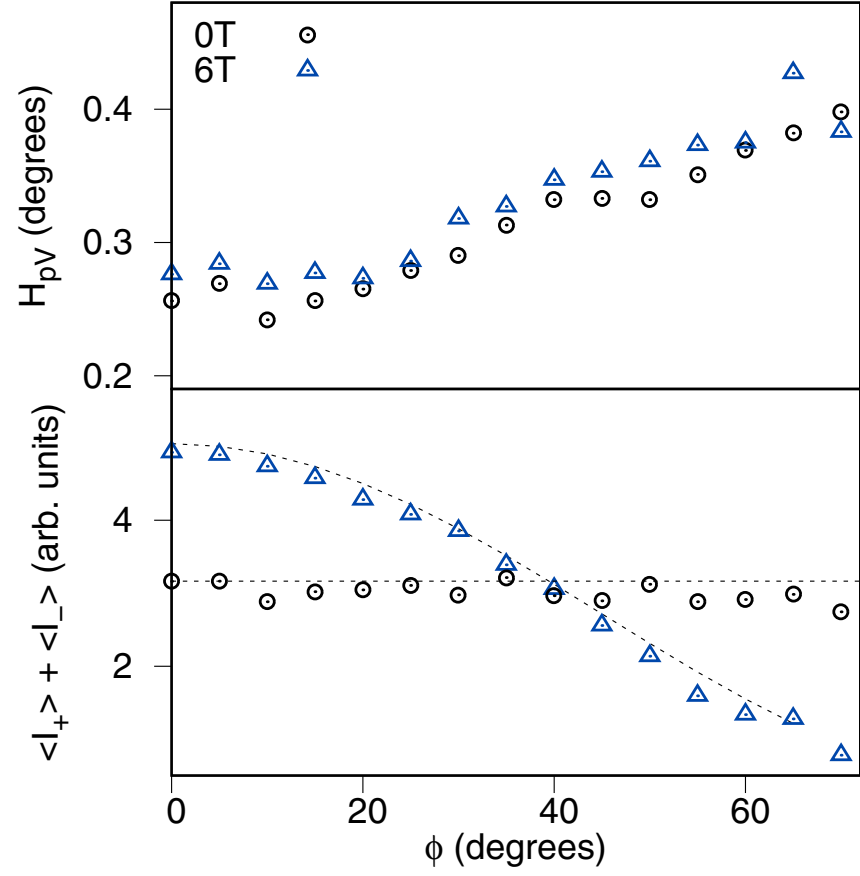

FIG. 3. The distribution of $H_{p V}$ and the sum of the integrated intensities for the reflection (111) measured with a magnetic field $6 \mathrm{~T}$ (triangles) and without it (circles) at the diffractometer $5 \mathrm{C} 1$ at $150 \mathrm{~K}$ over the Debye cone $\left(\phi=0^{\circ}\right.$ correspond to the scattering in the equatorial plane). The model values after 2D Rietveld refinement are given by the dotted lines.

error bars is observed for the 2D data. The refined parameters are also in good agreement with the literature: $-4.20(3) \mu_{B}$ and 3.97(3) $\mu_{B}$ [31].

Flipping sum and difference diffraction patterns measured at $150 \mathrm{~K}$ in a magnetic field of $6 \mathrm{~T}$ are shown in Fig. 4. The presence of magnetic scattering depending on the neutron spin orientation is clearly seen in the flipping difference pattern where reflections with a significant magnetic contribution are easily recognizable by the strong variation of their intensity along the Debye cone. The angular dependence of the integrated intensity of (111) reflection is shown in Fig. 3. It is in good agreement with the model values calculated by using formula (4) (dotted lines in Fig. 3). Note that the strong dependence of magnetic scattering on polar angle $\phi$ creates a problem in the reduction of the two-dimensional diffraction pattern to the one-dimensional one.

The use of polarized neutron diffraction improves considerably the precision of the determination of Fe magnetic moments. The values of magnetic moments obtained from the $2 \mathrm{D}$ refinement are $-4.03(1) \mu_{B}$ for the ion $\mathrm{Fe}^{3+}$ at the tetrahedral site and $3.95(1) \mu_{B}$ for that at the octahedral site. For the $1 \mathrm{D}$ data the moments are found to be $-4.05(7) \mu_{B}$ and $3.89(6) \mu_{B}$, respectively. Measurements performed below the Verwey transition did not show any evolution of the scattering signal (see Supplemental Material [23]). No new magnetic reflections associated with the ordering of octahedral $\mathrm{B}$ irons $\left(\mathrm{Fe}^{3+}, \mathrm{Fe}^{2+}\right)$ were observed, which is in agreement with previous polarized neutron single-crystal diffraction measurements [30]. 

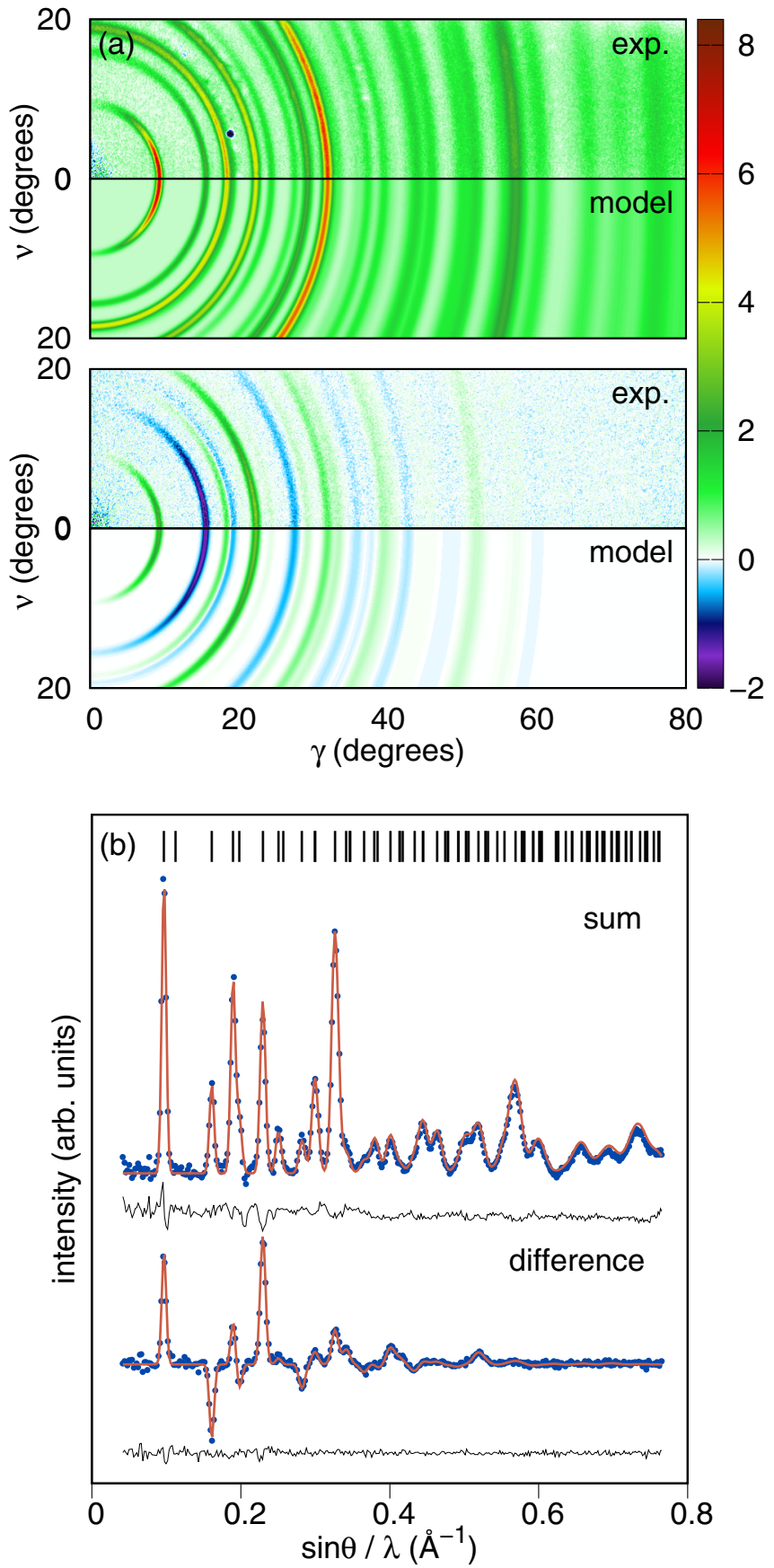

FIG. 4. Flipping sum and difference diffraction patterns collected on $\mathrm{Fe}_{3} \mathrm{O}_{4}$ at $T=150 \mathrm{~K}, 6 \mathrm{~T}$. The measured $2 \mathrm{D}$ pattern is shown on the top, the calculated one is shown on the bottom (a), and chi squares normalized per number of points is 3.93. Diffraction profiles estimated near the equatorial plane (b). The position of reflections is marked by "|." Black lines show the differences between experimental points (blue) and model line (orange).

\section{SPIN-ICE COMPOUND $\mathrm{Ho}_{2} \mathrm{Ti}_{2} \mathrm{O}_{7}$}

Among the rare-earth pyrochlores titanates $\mathrm{Ho}_{2} \mathrm{Ti}_{2} \mathrm{O}_{7}$ is considered as a canonical spin ice compound that shows various exotic magnetic states produced by the presence of geometric frustration [32,33]. It shows Ising-like behavior, with the magnetic moments being constrained along the local $\langle 111\rangle$ axes. In the pyrochlore lattice, the distinction between Ising, Heisenberg, or $X Y$ models cannot be based, as usual, on the analysis of the macroscopic properties of a singlecrystalline sample in a magnetic field because of the presence of four different anisotropy axes. The information about the local anisotropy of $\mathrm{Ho}_{2} \mathrm{Ti}_{2} \mathrm{O}_{7}$ has been first obtained by polarized neutron single-crystal diffraction based on the so-called "local susceptibility approach" [6]. The temperature behavior of the reported local susceptibility tensor has confirmed the Ising character of Ho local anisotropy and was in perfect agreement with that calculated from the rare-earth crystal field parameters. Here, we show that the same information about the local susceptibility tensor can be obtained by using $2 \mathrm{D} \mathrm{Ri}-$ etveld refinement of the polarized neutron powder diffraction patterns.

We collected a series of powder patterns from the $\mathrm{Ho}_{2} \mathrm{Ti}_{2} \mathrm{O}_{7}$ sintered powder sample in the temperature range $5-50 \mathrm{~K}$ and in a field of $1 \mathrm{~T}$. We found that applying a magnetic field to the sample led to dramatic changes in the diffraction pattern. As an example, the flipping diffraction patterns measured at $5 \mathrm{~K}$ in $1 \mathrm{~T}$ are shown in Fig. 5. As expected, a strong variation of intensity along the Debye cone is observed. It can be seen as well that the intensities of reflections allowed by $F d \overline{3} m$ symmetry $(111,220,113$, etc.) are strongly polarization dependent [see the flipping difference pattern in Fig. 5(b)]. We also found that the new reflections 200, 222, and 240 appear, which are forbidden by $F d \overline{3} m$ symmetry. As seen from the difference plot, the intensities of these reflections do not depend on neutron polarization but they are of purely magnetic origin. It has been shown that these reflections arise from the off-diagonal coefficient in the local susceptibility $\chi_{12}$ which becomes significant at low temperatures [13]. Note that the flipping difference pattern, proportional to $N \cdot M_{z, \perp}$, contains both positive and negative values, as its sign depends on the phase of the magnetic and nuclear structure factors.

For the space group $F d \overline{3} m$, the symmetry constraints imply that the local susceptibility tensor has only two independent matrix elements $\chi_{11}$ and $\chi_{12}$ and the principal axes of Ho magnetization ellipsoids are oriented along the four local $\langle 111\rangle$ axes. Their lengths, given by $\chi_{\|}=\chi_{11}+2 \chi_{12}$ and $\chi_{\perp}=\chi_{11}-\chi_{12}$, were determined at each temperature. The thermal evolution of $\chi_{\|}$and $\chi_{\perp}$ obtained by 2D Rietveld refinement on a polycrystalline sample is shown in Fig. 6 by solid symbols. Open symbols in the figure show the results of a previous study performed using polarized neutron diffraction on a single crystal [6]. One can see that the results of Rietveld refinement are in good agreement with the singlecrystal ones and offer the same precision of the susceptibility parameters.

\section{SINGLE-MOLECULE MAGNET: Co(II) COMPLEX}

The 2D Rietveld method is known to be a powerful tool allowing one to study powder samples having a preferred crystallite orientation [34]. Application of a magnetic field to anisotropic powder samples can induce the preferred crystallite orientation, as the net moment of the crystallites tends to align in the field direction. Since the resultant preferred orientation can be determined from the $2 \mathrm{D}$ patterns, one 

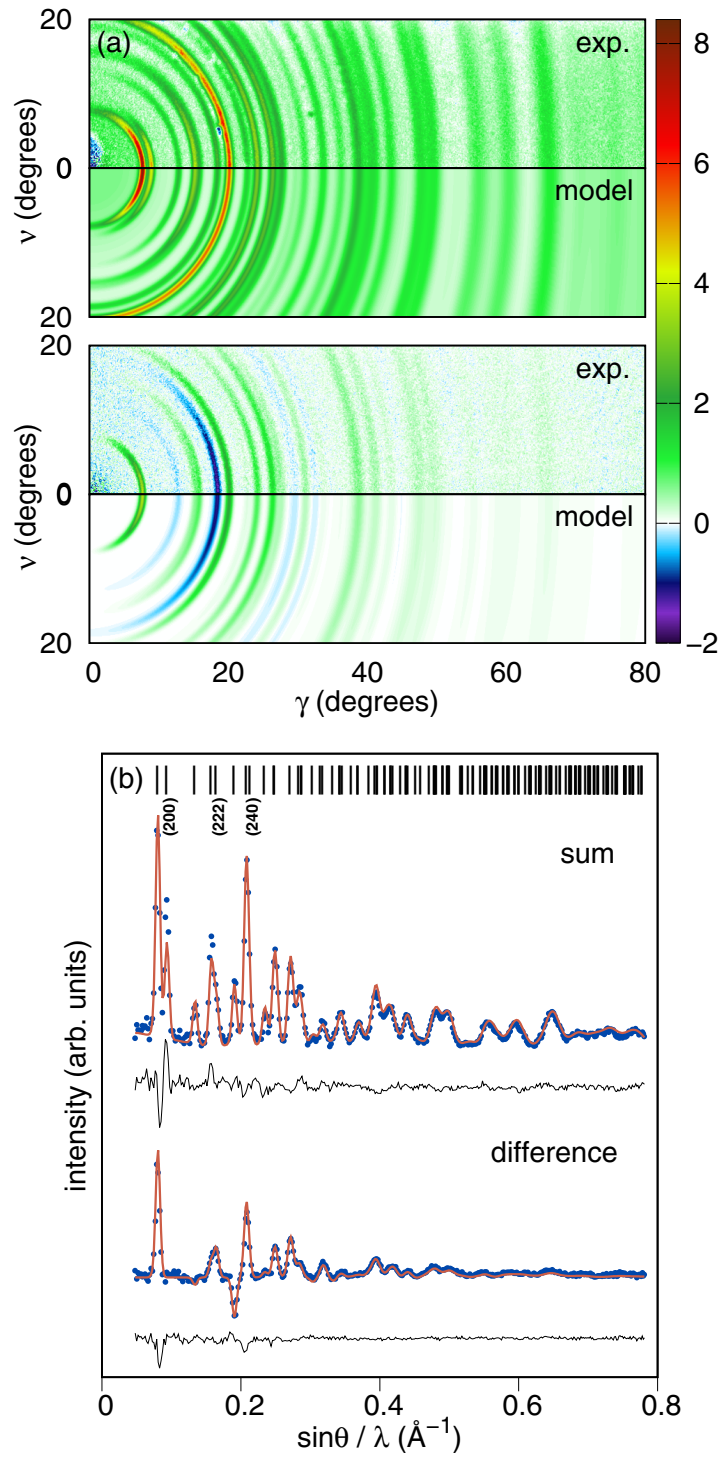

FIG. 5. The measured and calculated flipping sum (top) and difference (bottom) diffraction patterns collected on $\mathrm{Ho}_{2} \mathrm{Ti}_{2} \mathrm{O}_{7}$ at diffractometer $5 \mathrm{C} 1, T=5 \mathrm{~K}, H=1 \mathrm{~T}$ for (a) $2 \mathrm{D}$ and (b) $1 \mathrm{D}$ diffraction profiles. Chi squares normalized per number of points is 0.57 for $2 \mathrm{D}$ diffraction patterns.

can use these "magnetically textured" samples in PNPD. We found that such an approach in combination with the 2D Rietveld method has a number of advantages and we applied it to the studies of local susceptibility in the cobalt(II) complex with a molecular formula $\mathrm{Co}\left(\mathrm{L}_{1}\right)_{2} \mathrm{Cl}_{2}$, where $\mathrm{L}_{1}$ is tetramethylthiourea $\left[\left(\mathrm{CH}_{3}\right)_{2} \mathrm{~N}\right]_{2} \mathrm{CS}$ [35]. The compound is a single-molecule magnet that shows superparamagnetic behavior below a certain blocking temperature and exhibits magnetic hysteresis of purely molecular origin.

The powder was filled in a vanadium container of $6 \mathrm{~mm}$ diameter without compressing it. The sample was cooled to $2 \mathrm{~K}$ and the diffraction patterns were measured as a function of magnetic field. The Debye rings in zero field were found to be homogeneous, indicating the absence of preferred crystallite orientation. In magnetic fields above $1 \mathrm{~T}$ the crystallite reorientation started to appear and at $5 \mathrm{~T}$ the Debye rings were

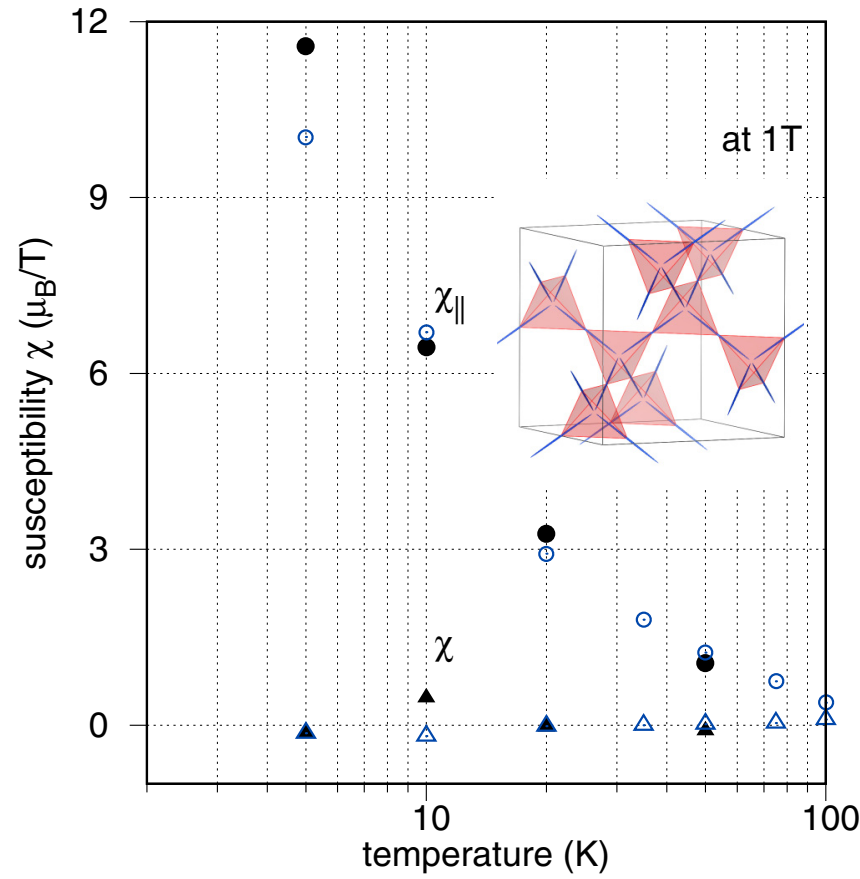

FIG. 6. Temperature dependence of the susceptibility components $\chi_{\|}$(circles) and $\chi_{\perp}$ (triangles) for single (open symbols, taken from Ref. [6]) and powder (solid symbols) $\mathrm{Ho}_{2} \mathrm{Ti}_{2} \mathrm{O}_{7}$ at $1 \mathrm{~T}$. The inset shows Ho magnetization ellipsoids at $50 \mathrm{~K}$.

transformed in a series of well-separated diffraction spots (Fig. 7). We note that the subsequent reduction of the magnetic field to $0 \mathrm{~T}$ did not change the crystallite orientation back to a random one. The diffraction patterns measured at $2 \mathrm{~K}$ and $5 \mathrm{~T}$ in the "magnetically textured" sample were used to determine the local susceptibility of the cobalt ion.

To take into account the preferred orientation we used a modified March model [36], which was developed to describe the mechanism of grain rotation that produces a preferred orientation,

$$
P_{h}=t+(1-t)\left[r^{2} \cos ^{2} \alpha_{h}+\frac{\sin ^{2} \alpha_{h}}{r}\right]^{-3 / 2},
$$

where $t$ is the fraction of randomly oriented crystallites, $\alpha_{h}$ is the angle between the transfer momentum and the preferred orientation axis, and $r$ describes the anisotropic shape of the crystallites. In the Debye-Scherrer geometry $r$ is more than one for platy crystallites and it is less than one for acicular crystallites. Although in our case the origin of preferred orientation is due to the application of a magnetic field, using the March distribution allows the estimation of an intuitively simple equivalent specimen compaction.

The studied single-molecule magnet has the monoclinic space group $P 2_{1} / n$ with $a=9.88 \AA, b=12.69 \AA, c=$ $14.13 \AA$, and $\beta=92.99^{\circ}$. It is composed of 43 atoms in the asymmetric unit [35], including 24 hydrogen atoms. The flipping patterns measured at $2 \mathrm{~K}$ and $5 \mathrm{~T}$ were used to refine the crystalline texture parameters and the susceptibility tensor of cobalt. As seen from Fig. 7, a very good agreement between patterns calculated after the refinement and the experimental ones is observed. Both the positions and the widths of the 


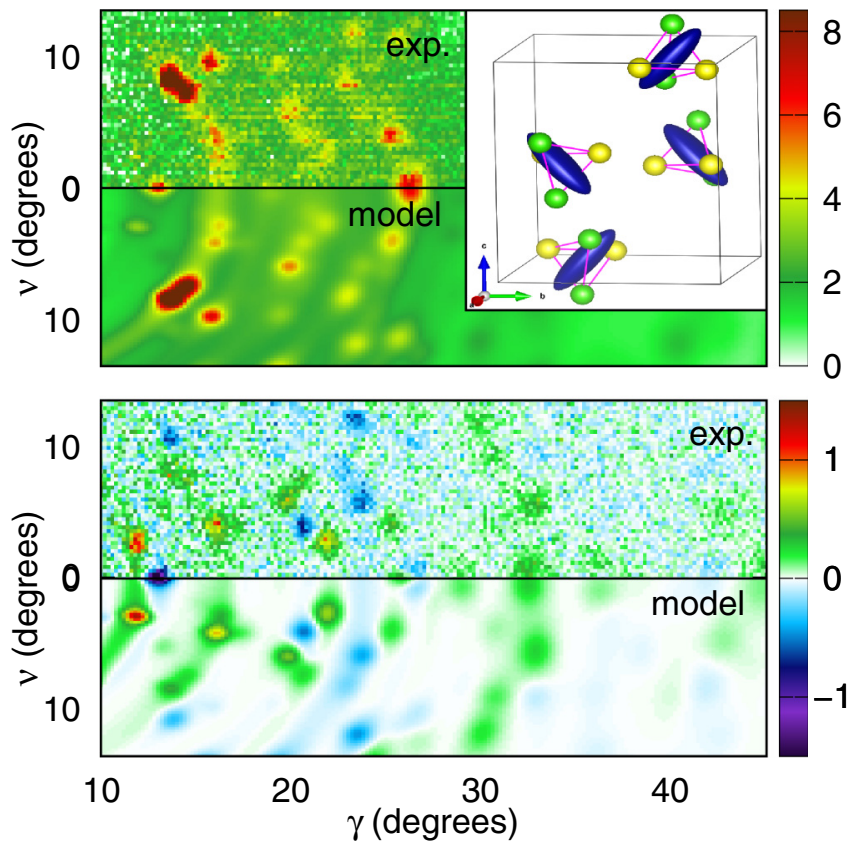

FIG. 7. The measured and calculated flipping sum (top) and difference (bottom) diffraction patterns collected on $\operatorname{Co}\left(\mathrm{L}_{1}\right)_{2} \mathrm{Cl}_{2}$ at diffractometer $6 \mathrm{~T} 2, T=2 \mathrm{~K}, H=5 \mathrm{~T}$. Chi squares normalized per number of points is 6.71. The inset shows Co magnetization ellipsoids surrounded by $\mathrm{S}$ (yellow) and $\mathrm{Cl}$ (green).

diffraction spots on the Debye cones are well reproduced in the model patterns. The refined March texture parameters $t=0.938(1)$ and $r=0.119(1)$ show that the magnetically induced preferred orientation is rather low at $5 \mathrm{~T}$ and only a small part of the crystallites is aligned with their easy axes parallel to the field. Hence, stronger fields are needed to overcome the steric hindrance in the powder packing. It is clear, however, that the presence of preferred orientation gives a big advantage in the 2D Rietveld refinement when using area detectors. As seen from the figure, different reflections with similar Bragg angles $2 \theta$ appear at different $\varphi$ angles. As a result, no overlapping of these reflections occurs, which allows one to use diffractometers with low resolution, such as the single-crystal diffractometer $6 \mathrm{~T} 2$, for powder diffraction. We note as well that the conventional approach consisting of the projection of 2D data on the 1D one for Rietveld refinement would result in a dramatic decrease of the resolution due to the reflection overlapping.

Finally, the refined magnetization tensor corresponding to an external field of $5 \mathrm{~T}$ for the cobalt atom in an asymmetric unit was found to be equal to

$$
\left(\begin{array}{ccc}
1.9(3) & 0.0(3) & 0.1(1) \\
0.0(3) & 2.3(3) & -1.4(2) \\
0.1(1) & -1.4(2) & 2.7(3)
\end{array}\right) \mu_{B} .
$$

The corresponding magnetization ellipsoid is presented in the inset of Fig. 7. The averaged magnetization estimated as 2.3(2) $\mu_{B}$ is close to the magnetization per cobalt atom $2 \mu_{B}$ taken from the magnetization measurements on a polycrystalline sample at $5 \mathrm{~T}$ [35]. A detailed analysis of the crystallite alignment, the evolution of the magnetization ellipsoids with temperature and field, as well as the theoretical interpretation of the ellipsoid orientation are still in progress and will be published later [37].

\section{CONCLUSION}

Our results suggest that the combination of an area detector, 2D Rietveld analysis, and the technique of magnetically induced preferred crystallite orientation opens direct route to the studies of local magnetic susceptibility in polycrystalline materials by polarized neutron diffraction. The results of the 2D Rietveld analysis of diffraction patterns from soft $\left(\mathrm{Fe}_{3} \mathrm{O}_{4}\right)$ and high $\left(\mathrm{Ho}_{2} \mathrm{Ti}_{2} \mathrm{O}_{7}\right)$ magnetic compounds are in perfect agreement with the single-crystal ones reported earlier. We demonstrate that using "magnetically textured" powder and the 2D Rietveld refinement allows us to obtain a precision in the determination of the susceptibility parameters close to that obtained in the single-crystal diffraction experiments. By applying this procedure to a single-molecule magnet in polycrystalline form, we obtained the local susceptibility tensor for a cobalt atom, which can now be compared with that predicted by the theory. More generally, we suggest that the magnetic structure determination by applying the 2D Rietveld method to the "magnetically textured" samples has significant perspectives, as it does not require a high instrumental resolution due to the fact that different types of reflections with similar Bragg angles are spread over the Debye cones.

\section{ACKNOWLEDGMENTS}

We are grateful to J. Overgaard for stimulating discussions and also providing us a single-molecule magnet with $\mathrm{Co}(\mathrm{II})$ complex. I.K. thanks CNRS for support through a postdoctoral position.
[1] B. Gillon and P. Becker, Modern Charge-Density Analysis (Springer, Dordrecht, 2012), pp. 277-302.

[2] J. Schweizer, Collection SFN 9, 87 (2008).

[3] A. Gukasov and P. J. Brown, J. Phys.: Condens. Matter 14, 8831 (2002).

[4] I. A. Kibalin, Z. Yan, A. B. Voufack, S. Gueddida, B. Gillon, A. Gukasov, F. Porcher, A. M. Bataille, F. Morini, N. Claiser,
M. Souhassou, C. Lecomte, J.-M. Gillet, M. Ito, K. Suzuki, H. Sakurai, Y. Sakurai, C. M. Hoffmann, and X. P. Wang, Phys. Rev. B 96, 054426 (2017).

[5] H. Cao, A. Gukasov, I. Mirebeau, P. Bonville, and G. Dhalenne, Phys. Rev. Lett. 101, 196402 (2008).

[6] H. Cao, A. Gukasov, I. Mirebeau, P. Bonville, C. Decorse, and G. Dhalenne, Phys. Rev. Lett. 103, 056402 (2009). 
[7] K. Ridier, B. Gillon, A. Gukasov, G. Chaboussant, A. Cousson, D. Luneau, A. Borta, J.-F. Jacquot, R. Checa, Y. Chiba, H. Sakiyama, and M. Mikuriya, Chem. - Eur. J. 22, 724 (2015).

[8] E. A. Klahn, C. Gao, B. Gillon, A. Gukasov, X. Fabrèges, R. O. Piltz, S.-D. Jiang, and J. Overgaard, Chem. - Eur. J. 24, 16576 (2018).

[9] E. Lelièvre-Berna, A. S. Wills, E. Bourgeat-Lami, A. Dee, T. Hansen, P. F. Henry, A. Poole, M. Thomas, X. Tonon, J. Torregrossa, K. H. Andersen, F. Bordenave, D. Jullien, P. Mouveau, B. Guérard, and G. Manzin, Meas. Sci. Technol. 21, 055106 (2010).

[10] A. Wills, E. Lelièvre-Berna, F. Tasset, J. Schweizer, and R. Ballou, Physica B 356, 254 (2005).

[11] H. Hiraka, K. Ohoyama, Y. Ogata, T. Ogawa, R. Gallage, N. Kobayashi, M. Takahashi, B. Gillon, A. Gukasov, and K. Yamada, Phys. Rev. B 90, 134427 (2014).

[12] O. Rivin, E. N. Caspi, H. Ettedgui, H. Shaked, and A. Gukasov, Phys. Rev. B 88, 054430 (2013).

[13] A. Gukasov and P. J. Brown, J. Phys.: Condens. Matter 22, 502201 (2010).

[14] P. Brown and J. Matthewman, The Cambridge Crystallography Subroutine Library, Rutherford Appleton Laboratory Report No. RAL-93-009, 1993 (unpublished).

[15] J. Rodriguez-Carvajal, Physica B 192, 55 (1993).

[16] A. C. Larson and R. B. Von Dreele, GSAS, General Structure Analysis System, Los Alamos National Laboratory Report No. LAUR-86-748, 1994 (unpublished).

[17] V. Petř́íček, M. Dušek, and L. Palatinus, Z. Kristallogr. - Cryst. Mater. 229, 345 (2014).

[18] F. Akeroyd, S. Ansell, S. Antony, O. Arnold, A. Bekasovs, J. Bilheux, J. Borreguero, K. Brown, A. Buts, S. Campbell, D. Champion, L. Chapon, M. Clarke, S. Cottrell, R. Dalgliesh, D. Dillow, M. Doucet, N. Draper, R. Fowler, M. A. Gigg et al., MANTID, http://dx.doi.org/10.5286/software/mantid (2013).

[19] P. Jacobs, A. Houben, W. Schweika, A. L. Tchougréeff, and R. Dronskowski, J. Appl. Crystallogr. 48, 1627 (2015).
[20] F. Kimura, W. Oshima, H. Matsumoto, H. Uekusa, K. Aburaya, M. Maeyama, and T. Kimura, CrystEngComm. 16, 6630 (2014).

[21] M. Stekiel, R. Przeniosło, M. Duczmal, D. Wardecki, and T. Buslaps, Cryst. Res. Technol. 50, 773 (2015).

[22] International Tables for Crystallography, Volume D, Physical Properties of Crystals, edited by A. Authier (Wiley, Hoboken, NJ, 2004).

[23] See Supplemental Material at http://link.aps.org/supplemental/ 10.1103/PhysRevResearch.1.033100 for details on the generalized expression for the powder averaging of $\left|\vec{M}_{\perp}\right|^{2}$ and $\left(\vec{M}_{\perp} \cdot \vec{P}\right)$ terms, the transformation matrix $\mathrm{T}$ definition, and the results of measurements on $\mathrm{Fe}_{3} \mathrm{O}_{4}$ performed below the Verwey transition.

[24] P. Norby, J. Appl. Crystallogr. 30, 21 (1997).

[25] The RHOCHI source code is written in PYTHON3 using the CRYSPY library. It is freely distributed through the GitHub service: https://github.com/ikibalin/cryspy, where a short guide of its application is given together with examples.

[26] W. C. Hamilton, Phys. Rev. 110, 1050 (1958).

[27] T. Okamura, Sci. Rep. Tohoku Imp. Univ. Ser. 1 21, 231 (1932).

[28] B. S. Ellefson and N. W. Taylor, J. Chem. Phys. 2, 58 (1934).

[29] E. J. W. Verwey, Nature (London) 144, 327 (1939).

[30] V. C. Rakhecha and N. S. Murthy, J. Phys. C: Solid State Phys. 11, 4389 (1978).

[31] J. P. Wright, J. P. Attfield, and P. G. Radaelli, Phys. Rev. B 66, 214422 (2002).

[32] M. J. Harris, S. T. Bramwell, D. F. McMorrow, T. Zeiske, and K. W. Godfrey, Phys. Rev. Lett. 79, 2554 (1997).

[33] J. S. Gardner, M. J. P. Gingras, and J. E. Greedan, Rev. Mod. Phys. 82, 53 (2010).

[34] M. Ferrari and L. Lutterotti, J. Appl. Phys. 76, 7246 (1994).

[35] S. Vaidya, P. Shukla, S. Tripathi, E. Rivière, T. Mallah, G. Rajaraman, and M. Shanmugam, Inorg. Chem. 57, 3371 (2018).

[36] W. A. Dollase, J. Appl. Crystallogr. 19, 267 (1986).

[37] J. Overgaard et al. (unpublished). 